\section{Synergie entre pompes à efflux}

\section{Rôle d'AcrAB-TolC dans I'initiation de la résistance aux antibiotiques}

Manuela Lizarralde-Guerrero, Florian Taraveau
École normale supérieure de Lyon,

Département de biologie, Master biologie, Lyon, France.

manuela.lizarralde-guerrero@ens-lyon.fr florian.taraveau@ens-lyon.fr

gènes tet $A$-tet $R$, puis la transcription et la traduction de ces gènes. Ils ont ainsi observé que la production de la pompe TetA chez les transconjugants est significativement plus élevée que chez les cellules donneuses. Ce résultat inattendu suggère que l'absence de TetR (inhibiteur de l'expression $\operatorname{de}$ tet $A$ ) dans la cellule receveuse donne lieu à la production incontrôlée de TetA, avant que TetR ne soit également produit et que l'inhibition ne se mette en place. Pour vérifier cela, le transfert de plasmide a été testé sur des cellules receveuses exprimant constitutivement TetR. La production de TetA après transfert du plasmide est alors totalement inhibée, ce qui vient confirmer l'hypothèse avancée. Cet effet, aussi nommé induction zygotique, est fréquent lors des transferts d'ADN entre bactéries [4]. L'absence d'inhibiteur chez la cellule receveuse permet alors l'expression rapide de l'ADN reçu.

En bref, le système ParB/parS permet de suivre le transfert conjugatif du plasmide contenant les gènes tet $A$-tet $R$. Chez les transconjugants, la production $\operatorname{de} T$ TetA est forte, suggérant que l'acquisition de la résistance à la tétracycline est bien effective.

En présence de tétracycline, la mise en place de la pompe TetA est ralentie mais reste possible

Une des questions essentielles abordée par les auteurs est l'étude des processus qui peuvent mener des bactéries receveuses, initialement sensibles à la tétracycline, à acquérir une résistance par conjugaison dans un milieu contenant déjà l'antibiotique. La tétracycline est un antibiotique dit bactériostatique, c'est-à-dire 


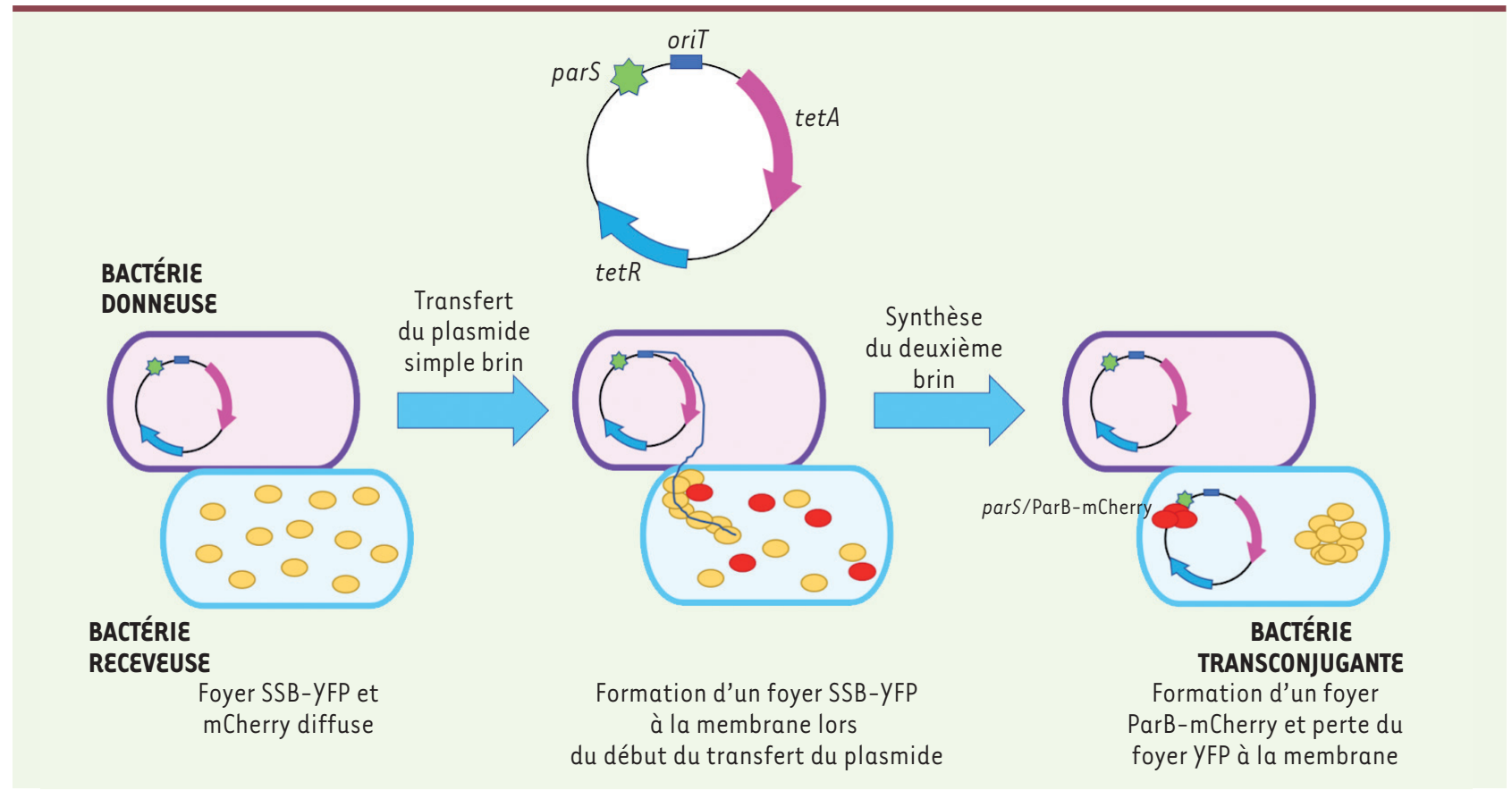

Figure 1. Schéma explicatif du système ParB/parS permettant de marquer en temps réel l'acquisition du plasmide conférant la résistance à la tétracycline. En jaune, YFP (marquant SSB). En rouge, mCherry (marquant ParB).

qui ne tue pas les bactéries mais inhibe leur croissance de manière réversible. La pompe à efflux TetA offre aux bactéries un mécanisme de résistance à la tétracycline en excrétant l'antibiotique hors de la cellule. Néanmoins, si la tétracycline est déjà présente dans la cellule, la mise en place de la pompe TetA pourrait être affectée. Pour étudier ce phénomène, les auteurs ont réalisé plusieurs séries de conjugaisons en présence ou en absence de tétracycline. La première observation rapportée est que la présence de tétracycline diminue fortement la production de TetA chez les transconjugants, sans l'empêcher complètement. Grâce au système de suivi de la conjugaison ParB/parS, il a pu être établi que la tétracycline n'influence pas le taux de réussite de la conjugaison. Une hypothèse peut alors être avancée en prenant en compte le mode d'action de la tétracycline. La tétracycline est un inhibiteur réversible de la traduction qui vient se fixer aux sous-unités ribosomiques [5] et peut donc, de cette manière, inhiber la traduction de toute nouvelle information génétique obtenue par la bactérie par transfert horizontal. Néanmoins, dans ce cas, pourquoi la présence de tétracycline ne suffit-elle pas à empêcher la traduction de TetA ? Une boucle positive d'activation pourrait être envisagée ici. Selon cette hypothèse, la production de quelques exemplaires de TetA permettrait d'exporter un peu de tétracycline hors de la cellule, laissant alors la possibilité de produire davantage $\operatorname{de}$ TetA et ainsi de lever progressivement l'inhibition. Néanmoins, grâce à l'utilisation de mutants de tetA $(\Delta t e t A)$, les auteurs ont montré que la pompe à efflux TetA n'était impliquée ni dans sa propre traduction, ni dans le maintien de la traduction dans l'ensemble de la cellule: chez les mutants $\Delta t e t A$, la traduction est toujours maintenue à un niveau basal en présence de tétracycline. La réponse se trouve donc chez un autre acteur moléculaire de l'arsenal d'Escherichia coli.

\section{Intervention de la pompe à efflux AcrAB-TolC dans l'acquisition de résistances aux antibiotiques en milieu défavorable}

Suite aux expériences précédentes, l'hypothèse de l'intervention d'une autre pompe à efflux au sein de la cellule a été avancée. Chez $\varepsilon$. coli, un tel complexe pro- téique existe et son rôle est déjà partiellement connu : la pompe AcrAB-TolC [6]. II s'agit d'une pompe à efflux non spécifique dont l'activité est finement régulée et qui prend en charge une très grande variété de xénobiotiques.

Afin d'analyser le rôle d'AcrAB-TolC, des mutants des différentes sous-unités du complexe ont été obtenus ( $\Delta a c r A, \Delta a c r B$, $\triangle$ tolC et des combinaisons des trois) et testés dans des conditions similaires à celles utilisées précédemment, en présence et en absence de tétracycline. Les résultats obtenus sont très clairs: lors de I'utilisation d'un mutant AcrAB-TolC (quel qu'il soit), la traduction, et donc la production de TetA, cesse complètement dans la cellule en présence de tétracycline. Cette action d'AcrAB-TolC a été étudiée de manière plus poussée par les auteurs, permettant de conclure que cette pompe à efflux, en participant à la sécrétion d'une partie de la tétracycline hors de la cellule bactérienne, permet de retarder et diminuer l'inhibition de la traduction. De cette manière, la production de TetA peut démarrer et l'acquisition de la résistance se met en place. 


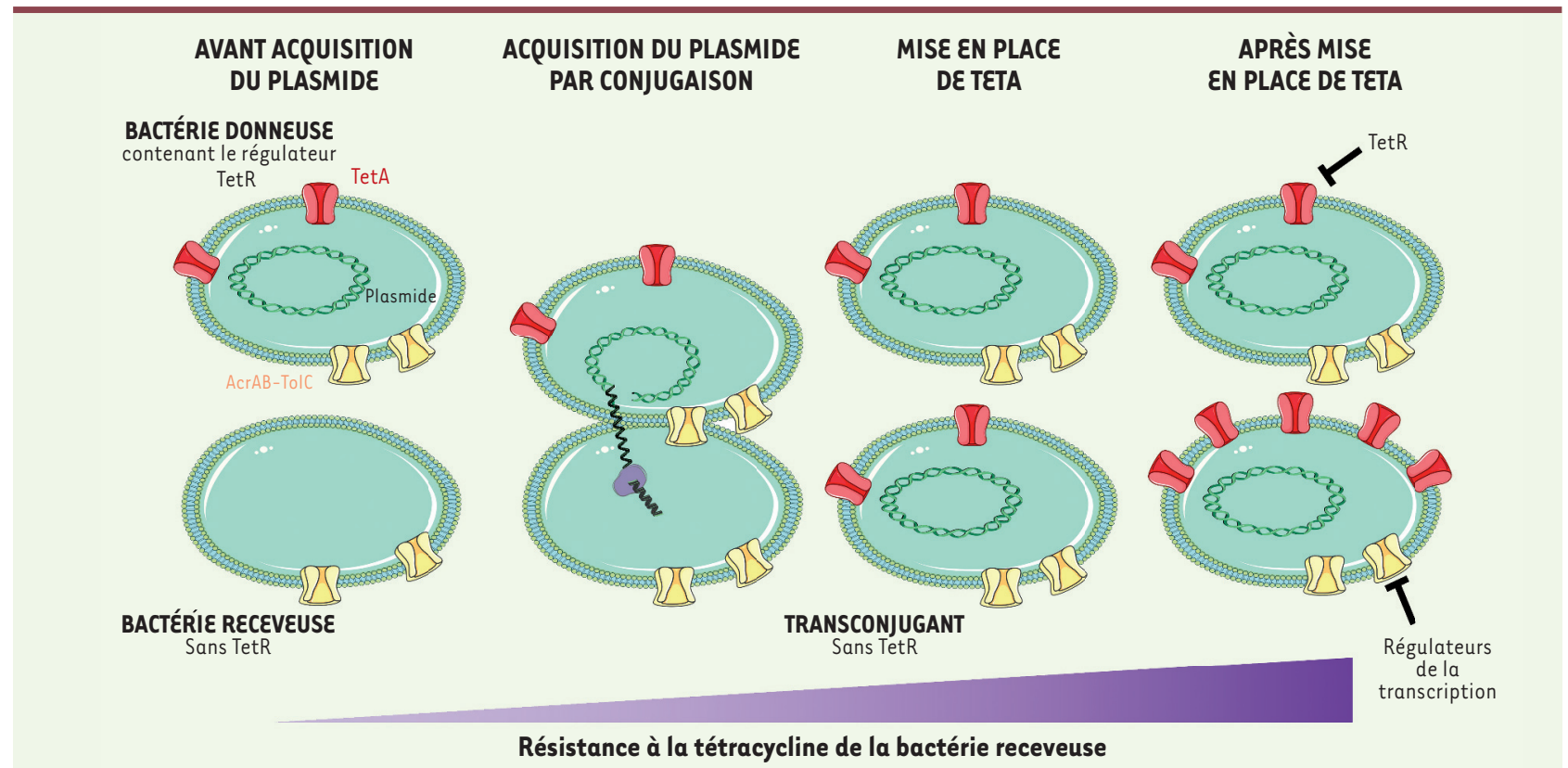

Figure 2. Les pompes à efflux AcrAB-TolC (jaune) et TetA (rouge) agissent ensemble pour éliminer la tétracycline hors de la cellule bactérienne. La production de TetA est inhibée par le répresseur TetR, tandis que celle de AcrAB-TolC est inhibée par différents régulateurs de la transcription ciblant au moins l'une des sous-unités de la pompe.

Il est finalement intéressant de noter que la production d'AcrAB-TolC est finement contrôlée, et est, en particulier, inhibée par des régulateurs de la transcription (Figure 2). Une mutation artificielle ciblant l'un de ces régulateurs provoque une augmentation de la quantité d'AcrAB-TolC, ce qui permet aux bactéries concernées d'éliminer rapidement des molécules antibiotiques. Cela faliciterait grandement l'apparition de résistances si une mutation semblable venait à se produire naturellement.

\section{Conclusion}

En conclusion, les transferts horizontaux permettent aux bactéries d'acquérir des résistances à une ou plusieurs molécules antibiotiques. Dans ce cadre, Nolivos et al montrent comment la pompe à efflux AcrAB-TolC facilite l'acquisition de ces résistances, comme c'est le cas avec la mise en place de la pompe TetA et l'acqui- sition de la résistance à la tétracycline. La concentration d'antibiotiques présente dans la bactérie est réduite par des pompes non spécifiques (AcrAB-TolC), permettant l'initiation de mécanismes de résistance spécifiques (pompe TetA). II est intéressant de noter qu'AcrAB-TolC est un système couramment retrouvé chez les bactéries multirésistantes aux antibiotiques [7]. Ce système reste spécifique aux bactéries du genre Escherichia mais des systèmes similaires existent chez de très nombreux genres bactériens. Dans son ensemble, cette étude permet d'élucider un nouveau mécanisme que les bactéries utilisent pour contourner l'action des antibiotiques, ouvrant également de nouvelles pistes pour combattre ces phénomènes d'acquisition de résistance à ces derniers. $\diamond$

Role of AcrAB-TolC multidrug efflux pump in drug-resistance acquisition by plasmid transfer

\section{LIENS D'INTÉRÊT}

Les auteurs déclarent n'avoir aucun lien d'intérêt concernant les données publiées dans cet article.

\section{RÉFÉRENCES}

1. Lerminiaux NA, Cameron ADS. Horizontal transfer of antibiotic resistance genes in clinical environments. Can J Microbiol 2019 ; 65 : 34-44.

2. Nolivos S, Cayron J, Dedieu A, et al. Role of AcrAB-TolC multidrug efflux pump in drug-resistance acquisition by plasmid transfer. Science $2019 ; 364: 778-82$.

3. Bignell C, Thomas CM. The bacterial ParA-ParB partitioning proteins. J Biotechnol $2001 ; 91: 1-34$

4. Feinstein SI, Low KB. Zygotic induction of the rac locus can cause cell death in $\varepsilon$. coli. Mol Gen Genet 1982 $187: 231-5$.

5. Nguyen F, Starosta AL, Arenz S, et al. Tetracycline antibiotics and resistance mechanisms. Biol Chem 2014 ; 395 : 559-75

6. Du D, Wang Z, James NR, et al. Structure of the AcrABTolC multidrug efflux pump. Nature $2014 ; 509: 512-5$

7. Chowdhury N, Suhani S, Purkaystha A, et al. Identification of AcrAB-TolC efflux pump genes and detection of mutation in efflux repressor AcrR from omeprazole responsive multidrug-resistant Escherichia coli isolates causing urinary tract infections. Microbiol Insights 2019; 12 : 1-10.

\section{Retrouvez toutes les Actualités de la Myologie sur les sites de :}

la Société Française de Myologie www.sfmyologie.org sfm la filière de santé neuromusculaire FILNEMUS www.filnemus.fr 